\title{
Clinical Usefulness of the Geriatric Depression Scale to Identify the Elderly at Risk of Suicide
}

\author{
Hyunsuk Jeong ${ }^{1}$, Beomwoo Nam², Sun-Jin Jo1, Won-Chul Lee ${ }^{1}$, and Hyeon Woo Yim ${ }^{1}$ \\ ${ }^{1}$ Department of Preventive Medicine, College of Medicine, The Catholic University of Korea, Seoul, Republic of Korea \\ ${ }^{2}$ Department of Psychiatry, School of Medicine, Konkuk University, Chungju, Republic of Korea
}

\begin{abstract}
Objective Population-based suicidal screening can be an important intervention method to reduce suicidal attempt rate in community. However, directly asking about suicidal behavior may be burdensome to non-mental health workers. This study aimed to evaluate the clinical usefulness of the Geriatric Depression Scale-15 (GDS-15) in identifying the elderly at risk of suicide in community.

Methods Nine hundred forty-eight over 60 years of age participated in this study. All participants completed the GDS-15. A trained interviewer interviewed each participant for suicidality including suicidal ideation, plan, and prior attempt using the Structured Clinical Interview for DSM-IV.

Results When the cut-off score of 10 in the GDS-15 was applied to identify the elderly at risk of suicide, the proportion of directly asking about suicidal behavior by non-mental health workers was reduced by $33.1 \%$; however, $19.5 \%$ at risk of suicide were missed. When the cut-off was changed to $6,100 \%$ at risk of suicide were covered by the GDS- 15 .

Conclusion Screening for suicidality using GDS-15 is a promising way to reduce the proportion of directly asking about suicidal behavior by non-mental health workers among the high-risk suicidal elderly in a community setting. Psychiatry Investig 2020;17(5):481-486
\end{abstract}

Key Words Elderly, Geriatric Depression Scale, Screening, Suicide.

\section{INTRODUCTION}

The suicide rate among the elderly is particularly high and rapidly increasing in Korea. In 2015, the suicide rate was 36.9 per 100,000 people in their 60 s, 62.5 per 100,000 people in their 70 s, and 83.7 per 100,000 people in their 80 s. $^{1}$

It is important to identify elderly people with suicidality in community settings and to take appropriate actions based on the severity of suicidality in order to reduce the suicidal death rate, since suicide prevention relies on timely and effective detection and treatment. The elderly showed higher proportion of fatal suicidal acts than young people. These observations indicate the importance of performing screening and assessment to identify seniors who have suicidal thoughts and im-

Received: November 8, 2019 Revised: February 13, 2020

Accepted: March 12, 2020

$\triangle$ Correspondence: Hyeon Woo Yim, MD, PhD

Department of Preventive Medicine, College of Medicine, The Catholic University of Korea, 222 Banpo-daero, Seocho-gu, Seoul 06591, Republic of Korea Tel: +82-2-2258-7860, Fax: +82-2-532-3820, E-mail: y1693@catholic.ac.kr

(c) This is an Open Access article distributed under the terms of the Creative Commons Attribution Non-Commercial License (https://creativecommons.org/licenses/by$\mathrm{nc} / 4.0$ ) which permits unrestricted non-commercial use, distribution, and reproduction in any medium, provided the original work is properly cited. plementing aggressive clinical interventions to treat them. Gatekeeper activities provided by non-mental health worker have been shown to be effective and promising strategies in prevention of suicide in community..$^{2-4}$ The finding that almost half of the elderly who committed suicide saw their primary care provider about a month before committing suicide provides a potential opportunity for their identification. ${ }^{5}$

However, the detection of suicidality is difficult in community settings not only because suicidal elderly often do not spontaneously report their thoughts of suicide, but also because non mental health workers who lack training in mental health often have difficulties asking the elderly about such sensitive issues as suicide. ${ }^{6}$

Conventionally, a two-stage screening is used to identify suicidality. That is, depression screening tools are administered first to the general population; then, if their scores are greater than the depression cut-off, they are examined further through in-depth questions to identify suicidality. Suicidal thoughts are often a symptom of depression and should always be taken seriously. A previous study showed that the 15-item Geriatric Depression Scale (GDS-15) accurately differentiated older primary care patients who had expressed sui- 
cidal ideation from those who had not, which suggested that these screenings might have effectively identified individuals for whom more in-depth suicide risk assessments were warranted. ${ }^{7}$ However, previous suicidal screening strategies have focused on identifying suicide ideation. ${ }^{7.8}$

The severity of suicidality exists along a continuum. It ranges from passive suicidal thoughts, such as thinking that one is better off dead, to active suicidal thoughts, such as thoughts of taking actions toward hurting oneself. From a clinical perspective, suicidal ideation alone does not necessarily lead to suicidal plans or attempts, but the formulation of suicidal plans or attempts reflects an increased intensity of one's desire to die. ${ }^{9}$ Therefore, people with suicidal plans and/or attempts should be assessed immediately, and their suicidal motivations and the seriousness of their suicidal thoughts should be examined by mental health professionals as well. ${ }^{10}$

This study was carried out for community suicide prevention projects by suicidal screening to find the people in suicide crisis among community suicidal high-risk groups and refer them to mental health professionals. Therefore, we conducted the suicidal screening for all Medicaid recipients over 60 year old living in a small city. The current research focused on the clinical usefulness of the GDS-15 as a suicidal screening tool before directly asking about suicidal behavior to identify the elderly at risk of suicide in community setting. We evaluated the proportion of directly asking questions about suicidal behavior by non-mental health workers and how many of the elderly at risk for suicide elderly was missed when applied with conventional depression cut-off of the GDS-15 in a community setting.

\section{METHODS}

\section{Participants}

The source population was all Medicaid recipients over 60 years of age in Chungju city, a small town located in the central part of South Korea. The whole population of the target area was 208,202. Among them 60 or older were 40,196 (19.3\%). Among them, 1,088 were classified as Medicaid recipients in 2013. Of 1,088 elderly, 948 (87.1\%) participated in the study. Ninety-two elderly refused to participate and 48 were excluded because they could not communicate due to hearing loss or dementia. The surveys were conducted between July and October 2013. Chungju city is one of the regions with high suicide rates in Korea. The suicide rate was 36.5 per 100,000 for the whole population and 72.1 for elderly people over 60 years old in Chungju City while the Korean nationwide suicide rate was 28.5 per 100,000 in 2013. There were no statistical differences between participants and nonparticipants in age and sex. All of the elderly participants were recipients of Medicaid, or home care nursing services, including the management care of chronic physical diseases (e.g., hypertension, diabetes, etc.), and those who had regularly received financial aids from community health care centers. Informed consents were acquired from all participants following explanation of the nature of the principles of research, including confidentiality and the freedom of choice to participate. This study received a full review and an approval from the Institutional Review Board of The Catholic University of Korea (CUMC11U035). All procedures were in accordance with the Helsinki Declaration.

\section{Measurements}

\section{Socio-demographic characteristics}

Socio-demographic characteristics, including age, sex, years of education, and living situation (e.g., living alone, living with others) were recorded.

\section{Geriatric Depressive Scale-15}

Depressive symptoms were assessed by self-report with a visiting nurse assisted to read the questionnaire using the Korean version of the 15 -item GDS (score range $0-15$ ) with the cut-off score of $10 .^{11}$ The GDS-15 is consisted of 15 items in a yes/no format and a total score of zero to 15 . Higher score indicates more severe depressive symptoms. Internal consistency was assessed using Cronbach's alpha and was found to be 0.93 . The Korean version of GDS-15 was found to be an efficient and effective instrument with a good internal consistency reliability, content and discriminant validity for screening depression among the elderly. ${ }^{12}$

\section{Structured Clinical Interview for DSM-IV (SCID)}

The presence of suicidal ideation, suicide plans, and suicide attempts was determined by trained interviewers using the Structured Clinical Interview for DSM-IV Axis I Disorders (SCID-I), which was the gold standard for this assessment.: 'Have you seriously thought about committing suicide during the past month?'; 'Have you made a plan for committing suicide during the past month?'; and 'Have you attempted suicide during the past month?' The reference time period used one month to calculate the presence of suicidality because current is defined as "during the past month" for suicidality diagnosis. ${ }^{13}$ The SCID is a structured symptom-focused diagnostic interview that involves asking research participants direct questions. On the other hand, clinician's diagnostic interview relies not only on direct questions about symptoms but also on multiple other factors including observing patients' nonverbal expressions and listening to their life time stories. The findings suggest that clinician's diagnostic interviews provide 
comparable psychometric properties to the SCID regarding it as the "gold standard" assessment. ${ }^{14}$ The SCID is commonly considered to be the gold standard in diagnosing psychiatric conditions because it is representative of the DSM-IV. Some studies used suicidal questionnaires of SCID to determine a presence of suicidality. ${ }^{15,16}$ Korean version of SCID was revealed moderate to excellent inter-rater agreement and recommended as an accurate diagnostic tool in clinical practice and research on psychiatric disorders. ${ }^{17}$ We categorized the presence or absence of suicide ideation, plans, and attempts based on the subject response pattern (i.e., "yes" or "no"). The prevalence of suicidal ideation, suicide plans, and suicide attempts was computed. "At risk of suicide" was defined as the presence of suicide plan or suicide attempt during the past month. To ensure reliability, the interviewers were trained for interviewing techniques at a 2-day long (16-hour) workshop before being put in practice. They were kept blind to the results of the GDS-15 scores.

\section{Statistical analysis}

Demographic and clinical characteristics were summarized using the descriptive statistics such as numbers and percentages for categorical variables or means and SDs for continuous variables. The receiver operating characteristic (ROC) curve analysis of the GDS for identifying riskiness of suicide was evaluated by estimating sensitivity and specificity at different cut-off points. We simulated to see the effect of adjusting cutoff score until it covered $100 \%$ of the elderly at risk of suicide, even though 10 was the conventional depression cutoff score in the GDS validated for Korean population. We also examined how the proportion of the interviewers' directly asking about suicidal behavior changed according to each cut off. Accuracy was measured using the area under the curve (AUC) of the ROC. All analyses were conducted using the software package SAS version 9.3 (SAS Institute Inc., Cary, NC, USA) and MedCalc 12.7.0 (MedCalc Software, Ostend, Belgium).

\section{RESULTS}

Demographic characteristics of the 948 participants are provided in Table 1; 70.6\% were female, $47.4 \%$ were aged 75 years or older, $68.2 \%$ had $0-6$ years of educational attainment, and $51.7 \%$ were living alone (Table 1 ).

Of the 948 total participants, 314 (33.1\%) were positive for depressive symptoms (GDS-15 $\geq 10), 92$ (9.7\%) had had suicidal ideations, 40 (4.2\%) had made a suicidal plan, and 8 $(0.8 \%)$ had made a suicide attempt during the past month interviewed with SCID. Among them, 41 classified as being at risk of suicide (suicide plan only, $n=33$; suicide attempt only, $\mathrm{n}=1$; both suicide plan and attempt, $\mathrm{n}=7$ ). When a cut-off score
Table 1. Demographic characteristics of the 948 participants

\begin{tabular}{lc}
\hline \multicolumn{1}{c}{ Variables } & $\mathrm{N}(\%)$ \\
\hline $\begin{array}{l}\text { Age (years) } \\
60-74\end{array}$ & $499(52.6)$ \\
$\geq 75$ & $449(47.4)$ \\
Sex & \\
$\quad$ Male & $279(29.4)$ \\
Female & $669(70.6)$ \\
Educational attainment & \\
$0-6$ & $408(68.2)$ \\
$\geq 7$ & $190(31.8)$ \\
Living alone & \\
Yes & $490(51.7)$ \\
No & $458(48.3)$ \\
\hline
\end{tabular}

of GDS-15 $\geq 10$ was applied to identify at risk for suicide, the proportion of the interviewers' directly asking about suicidal behavior was reduced by $33.1 \%$; however, 8 (19.5\%) out of the 41 elderly participants who were identified as being at risk of suicide were still missed. When the cut-off of 6 was applied, $100 \%$ of the elderly at risk of suicide were detected through the GDS-15 screening, and the proportion of the interviewers' directly asking about suicidal behavior in was reduced by only $36.0 \%$ compared to when not using the GDS-15 (Table 2).

ROC curves were used to assess the usefullness of the GDS15 in discriminating between people who were at risk of suicide and those who were not. To use the GDS- 15 for detecting the riskiness of suicide, the AUC was 0.83 (95\% CI: 0.81-0.86) (Figure 1).

\section{DISCUSSION}

The current study showed that the proportion of directly asking about suicidal behavior by non-mental health workers was reduced by $33.1 \%$ when the conventional depressive symptom cut off score of 10 or greater was applied to identify the elderly at risk of suicide. However, 8 (19.5\%) out of 41 at risk of suicide were missed. When the cut-off was changed to 6 or more, $100 \%$ of them were covered by the GDS-15 screening.

A person's suicidality seems to change considerably over time; therefore, the lifetime or one-year prevalence may not accurately reflect immediate suicidal behavior. The reference time period used to calculate the prevalence of a condition (such as a month or a year) depends on the chronicity or stability of the target disease. One month, rather than one year, may be a better reference period for estimating the prevalence of suicidality to prevent suicide deaths. Due to the fluctuating nature of suicidality, it is hard to predict the actual point at 
Table 2. Clinical usefulness of the GDS-15 to identify risk of suicide

\begin{tabular}{|c|c|c|c|c|c|}
\hline \multirow{2}{*}{$\begin{array}{l}\text { GDS-15 } \\
\text { scores }\end{array}$} & \multicolumn{3}{|c|}{ Numbers of subjects $(\mathrm{N})$} & \multicolumn{2}{|c|}{ Clinical usefulness parameters (\%) } \\
\hline & Participants & $\begin{array}{c}\text { Detected at } \\
\text { risk of suicide* }\end{array}$ & $\begin{array}{l}\text { Missed at risk } \\
\text { of suicide* }\end{array}$ & $\begin{array}{c}\text { Decreased direct } \\
\text { asking rates for suicide }\end{array}$ & $\begin{array}{l}\text { Detection rate of } \\
\text { at risk of suicide }\end{array}$ \\
\hline 15 & 25 & 5 & 36 & 97.4 & 12.2 \\
\hline$\geq 14$ & 70 & 12 & 29 & 92.6 & 29.3 \\
\hline$\geq 13$ & 129 & 23 & 18 & 86.4 & 56.1 \\
\hline$\geq 12$ & 196 & 29 & 12 & 79.3 & 70.7 \\
\hline$\geq 11$ & 254 & 31 & 10 & 73.2 & 75.6 \\
\hline$\geq 10^{\dagger}$ & 314 & 33 & 8 & 66.9 & 80.5 \\
\hline$\geq 9$ & 382 & 37 & 4 & 59.7 & 90.2 \\
\hline$\geq 8$ & 463 & 39 & 2 & 51.2 & 95.1 \\
\hline$\geq 7$ & 530 & 39 & 2 & 44.1 & 95.1 \\
\hline$\geq 6$ & 607 & 41 & 0 & 36.0 & 100.0 \\
\hline$\geq 5$ & 679 & 41 & 0 & 28.4 & 100.0 \\
\hline$\geq 4$ & 745 & 41 & 0 & 21.4 & 100.0 \\
\hline$\geq 3$ & 810 & 41 & 0 & 14.6 & 100.0 \\
\hline$\geq 2$ & 864 & 41 & 0 & 8.9 & 100.0 \\
\hline$\geq 1$ & 900 & 41 & 0 & 5.1 & 100.0 \\
\hline$\geq 0$ & 948 & 41 & 0 & 0.0 & 100.0 \\
\hline
\end{tabular}

*at risk of suicide defined as among those with suicidality, anyone who had planned or attempted suicide, ${ }^{\dagger}$ depression cut-off score. GDS-15: Geriatric Depression Scale-15

which the actions occur or the thoughts are processed. ${ }^{18}$

The GDS-15 cut-off score to detect depression among the elderly in Korea is much higher than those in other Western and Eastern countries. The cut-off score was reported to be 5 points in Japan, ${ }^{19}$ Brazil,${ }^{20}$ and Canada, ${ }^{21}$ but 10 points in Korea. ${ }^{11}$ Because GDS-15 cut-off scores for depression vary from country to country, it is necessary to use country-specific cutoffs to evaluate the clinical usefulness of labeling at risk for suicide.

A breakthrough was made by Heisel et al. ${ }^{7}$ who investigated the effectiveness of the GDS in detecting suicide ideation. Geriatrics Depression Scale-Suicide Ideation (GDS-SI) is 5 subscale of GDS. A score of 1 or more on the five items of the GDSSI considered "at risk of suicide." Furthermore, a previous study suggested that both the 4 - and the 5-item versions are excellent alternatives to the 15-item version of GDS, and all of them are reasonable tools for detecting a presence of suicide ideation. ${ }^{22}$ However, we tried to detect suicide risk by adjusting the cut-off using the GDS-15 total score, because a score of 1 or more on GDS-SI was $87.6 \%$ in the current study. When a cut-off score of 10 was applied in the first stage of the screening process to identify riskiness of suicide, the proportion of health care professionals asking participants directly asking about their suicidal behavior was reduced by $33.1 \%$; however,

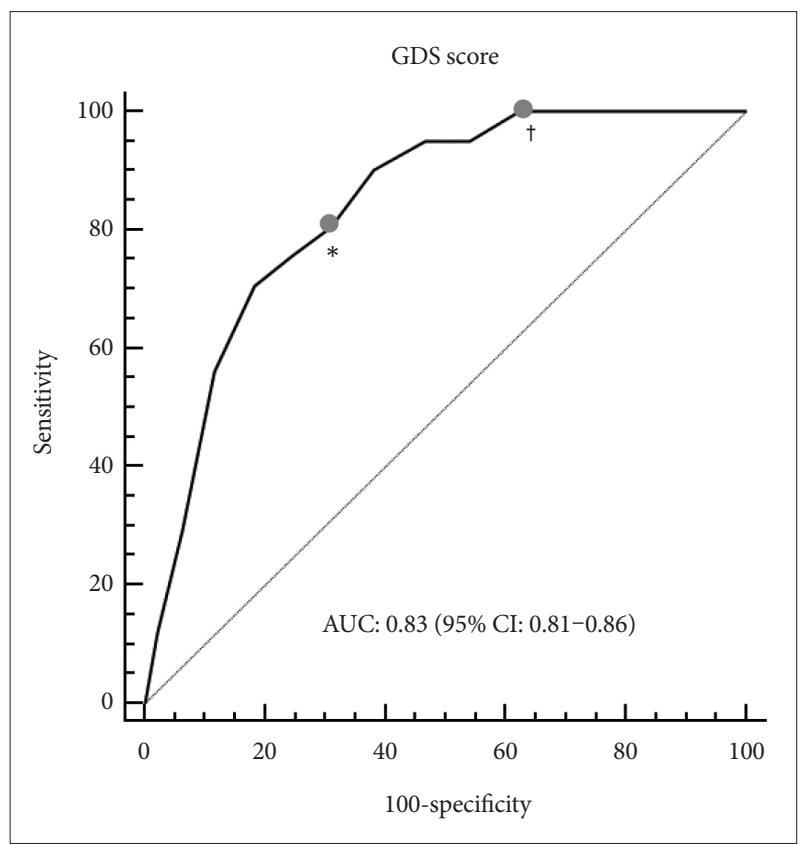

Figure 1. The Receiver Operating Characteristic (ROC) curves of the performance of the two-stage screening strategy in discriminating between participants at risk for suicide and those who are not. At risk for suicide refers to anyone who had planned or attempted suicide. *depression cut-off point (10), ${ }^{\dagger}$ cut-off point included $100 \%$ of those in at risk for suicide (6). GDS: Geriatric Depression Scale (GDS-15), AUC: area under the curve. 
8 (19.5\%) out of 41 elderly participants at risk of suicide were missed. When the cut-off was changed to $6,100 \%$ of the participants at risk of suicide were detected through the GDS-15 screening, and the proportion of the healthcare professionals' directly asking about suicidal behavior was reduced by $36.0 \%$ in our data.

The AUC value of the GDS-15 was 0.83 in the present study indicating that the GDS-15 can discriminate between people who were at risk of suicide and those who were not. The AUC $\geq 0.70$, suggesting fair or better discrimination between patients with and without suicide or suicide attempts. ${ }^{23} \mathrm{Com}-$ pared to conventional suicide risk screening tools such as SAD PERSONS (AUC $=0.58$ ) ${ }^{24}$ and Suicide Trigger Scale (AUC= $0.72),{ }^{25}$ GDS-15 can be used as a screening tool for finding suicide risk in elderly people.

A previous study reported that many elderly people at risk of suicide exhibited a subclinical depressive state rather than major or minor depression. ${ }^{26}$ When the cut-off value for detecting riskiness of suicide was set at a point a little below to the cut-off value for detecting depression, $100 \%$ of the elderly at risk of suicide was covered in the present study. This might have been an accidental over-estimated result in our study but it seems to be clear that people at risk of suicide show subclinical depression symptoms and based on that, the studies for finding appropriate cut-offs for detecting the risk of suicide using other national or ethnic populations are in order.

It is well known that more than $90 \%$ people who commit suicide suffer from psychiatric disorders. Among the elderly, depression is particularly common. According to the US Preventive Services Task Force guideline, ${ }^{27}$ suicidal screening was recommended primarily to high-risk populations, such as those with depression or other mental health issues. The present findings highlight the potential screening method for late-life suicidality with a brief depression scale in community settings for high risk group considering prevalence of depression. The fact that a cut-off score of 6 in the GDS-15 produced 100\% sensitivity for detecting the risk of suicide suggests a possible candidate for more in-depth assessment of suicidal risk which might be greatly helpful for high suicidal risk individuals and for primary care clinics as well.

These results should be considered in the context of some potential limitations. We obtained our data only from elderly Medicaid recipients who comprised a group of individuals with a high risk of suicidality in the community. This should be taken into account when generalizing the results to other population. Because suicidal behaviors are related to various forms of social interaction including social isolation, feelings of loneliness, poor social support, lack of closed persons, and socioeconomic disadvantage. A previous report suggested that low socioeconomic status was associated with an increased risk of suicidal ideation among the elderly. ${ }^{28}$

The one-month prevalence of suicide risk was $4.3 \%$ in the present data. According to a 2011 psychiatric epidemiology survey in Korea, the one-month prevalence of suicide risk was $0.4 \%$ in the general population. ${ }^{29}$ In our study there were only 8 suicide attempters during the past month. However, a cross nation studies reported that risk of suicide plans and attempts also was highest when suicidal ideation had within the first year of ideation. Remarkably, $60 \%$ of the transitions from ideation to attempt-as well as from ideation to plan and plan to attempt-occur within the first year of onset of ideation. ${ }^{30}$ Therefore, it cannot be overlooked people with suicidal ideation or plan.

Given the rarity of suicide, finding an accurate screening strategy for suicide risk for the general population is a challenge. However, previous studies reported that suicidal screening was effective among those who have high prevalence of suicidality, such as low income elderly and primary care patients. ${ }^{8}$ This study highlighted that Medicaid recipients might be an appropriate population for suicidality screening.

An important implication of this study is that screening for suicidality using this two-stage screening protocol using GDS15 is a promising way to reduce the proportion of directly asking about suicidal behavior by non-mental health workers among the high-risk suicidal elderly in a community setting. In future studies, appropriate GDS-15 cut-off points need to be explored among other regional and ethnic populations to identify $100 \%$ of the elderly at risk of suicide in community. The clinical and economic benefits of suicidal screening for high-risk people using the GDS-15 need to be further evaluated and investigated.

\section{Acknowledgments}

This study was supported by a grant of the Korea Mental Health Technology R\&D Project, Ministry of Health \& Welfare, the Republic of Korea (HM18C2235). The Ministry of Health and Welfare had no further role in study design; in the collection, analysis, and interpretation of the data; in the writing of the paper; or in the decision to submit the paper for publication.

\section{Conflicts of Interest}

The authors have no potential conflicts of interest to disclose.

\section{Author Contributions}

Conceptualization: Hyunsuk Jeong, Hyeon Woo Yim. Data curation: Hyunsuk Jeong, Beomwoo Nam, Sun-Jin Jo, Hyeon Woo Yim. Formal analysis: Hyunsuk Jeong, Hyeon Woo Yim. Funding acquisition: Hyeon Woo Yim. Investigation: all authors. Methodology: Hyunsuk Jeong, Won-Chul Lee, Hyeon Woo Yim. Project administration: Hyunsuk Jeong, Beomwoo Nam, Sun-Jin Jo. Resources: Hyunsuk Jeong, Hyena Woo Yim. Software: Hyunsuk Jeong. Supervision: Hyeon Woo Yim. Validation: Won-Chul Lee, Hyeon Woo Yim. Visualization: Hyunsuk Jeong, Hyeon Woo Yim. Writing—original draft: Hyunsuk Jeong. Writing_-review \& editing: all authors.

\section{ORCID iDs}

Hyunsuk Jeong Thtps:/orcid.org/0000-0001-5274-3816 
Beomwoo Nam https://orcid.org/0000-0003-3530-1746 Sun-Jin Jo https://orcid.org/0000-0002-8465-9632 Won-Chul Lee https://orcid.org/0000-0001-8052-2420

Hyeon Woo Yim https://orcid.org/0000-0002-3646-8161

\section{REFERENCES}

1. Korean National Statistics. Available at: http://www.nso.go.kr. 2016. Accessed December 1, 2018.

2. Zalsman G, Hawton K, Wasserman D, van Heeringen $\mathrm{K}$, Arensman E, Sarchiapone M, et al. Suicide prevention strategies revisited: 10-year systematic review. Lancet Psychiatry 2016;3:646-659.

3. Coppens E, Van Audenhove C, Iddi S, Arensman E, Gottlebe K, Koburger $\mathrm{N}$, et al. Effectiveness of community facilitator training in improving knowledge, attitudes, and confidence in relation to depression and suicidal behavior: results of the OSPI-Europe intervention in four European countries. J Affect Disord 2014;165:142-150.

4. Terpstra S, Beekman A, Abbing J, Jaken S, Steendam M, Gilissen R. Suicide prevention gatekeeper training in the Netherlands improves gatekeepers' knowledge of suicide prevention and their confidence to discuss suicidality, an observational study. BMC Public Health 2018;18:637.

5. Luoma JB, Martin CE, Pearson JL. Contact with mental health and primary care providers before suicide: a review of the evidence. Am J Psychiatry 2002;159:909-916.

6. Goldberg DP, Gask L, Zakroyeva A, Proselkova E, Ryzhkova N, Williams P. Training teachers to teach mental health skills to staff in primary care settings in a vast, under-populated area. Ment Health Fam Med 2012;9:219-224.

7. Heisel MJ, Duberstein PR, Lyness JM, Feldman MD. Screening for suicide ideation among older primary care patients. J Am Board Fam Med 2010;23:260-269.

8. Alexopoulos GS, Reynolds CF 3rd, Bruce ML, Katz IR, Raue PJ, Mulsant $\mathrm{BH}$, et al. Reducing suicidal ideation and depression in older primary care patients: 24-month outcomes of the PROSPECT study. Am J Psychiatry 2009;166:882-890.

9. Greenhill LL, Waslick B. Management of suicidal behavior in children and adolescents. Psychiatr Clin North Am 1997;20:641-666.

10. Mental Health and Drug and Alcohol Office. Mental Health for Emergency Departments-A Reference Guide. Sydney: NSW Department of Health; 2009.

11. Cho MJ, Suh GH, Hahn BJ, Kim HK, Lee DW, Kang MH. Validation of geriatric depression scale, Korean version (GDS) in the assessment of DSM-III-R major depression. J Korean Neuropsychiatr Assoc 1999;38: 48-63.

12. Bae JN, Cho MJ. Development of the Korean version of the Geriatric Depression Scale and its short form among elderly psychiatric patients. J Psychosom Res 2004;57:297-305.

13. Sheehan DV, Lecrubier Y, Sheehan KH, Amorim P, Janavs J, Weiller E, et al. The Mini-International Neuropsychiatric Interview (M.I.N.I.): the development and validation of a structured diagnostic psychiatric interview for DSM-IV and ICD-10. J Clin Psychiatry 1998;59(Suppl 20): 22-33;quiz 34-57.

14. Drill R, Nakash O, DeFife JA, Westen D. Assessment of clinical information: Comparison of the validity of a Structured Clinical Interview (the SCID) and the Clinical Diagnostic Interview. J Nerv Ment Dis 2015; 203:459-462.

15. Altura KC, Patten SB, Fiest KM, Atta C, Bulloch AG, Jette N. Suicidal ideation in persons with neurological conditions: prevalence, associations and validation of the PHQ-9 for suicidal ideation. Gen Hosp Psychiatry 2016;42:22-26.

16. Uebelacker LA, German NM, Gaudiano BA, Miller IW. Patient health questionnaire depression scale as a suicide screening instrument in depressed primary care patients: a cross-sectional study. Prim Care Companion CNS Disord 2011;13.

17. Hahn OS, Ahn JH, Song SH, Cho MJ, Kim JK, Bae JN, et al. Development of Korean version of structured clinical interview schedule for DSM-IV axis I disorder: interrater reliability. J Korean Neuropsychiatr Assoc 2000;39:362-372.

18. Nock MK, Kessler RC. Prevalence of and risk factors for suicide attempts versus suicide gestures: analysis of the National Comorbidity Survey. J Abnorm Psychol 2006;115:616-623.

19. Schreiner AS, Hayakawa H, Morimoto T, Kakuma T. Screening for late life depression: cut-off scores for the Geriatric Depression Scale and the Cornell Scale for Depression in Dementia among Japanese subjects. Int J Geriatr Psychiatry 2003;18:498-505.

20. Paradela EM, Lourenco RA, Veras RP. [Validation of geriatric depression scale in a general outpatient clinic]. Rev Saude Publica 2005;39:918923.

21. Herrmann N, Mittman N, Silver IL, Shulman KI, Busto UA, Shear NH, et al. Practice Guideline for the Assessment and Treatment of Patients With Suicidal Behaviors. Washington, DC: American Psychiatric Assoication; 2003.

22. Cheng ST, Yu EC, Lee SY, Wong JY, Lau KH, Chan LK, et al. The geriatric depression scale as a screening tool for depression and suicide ideation: a replication and extention. Am J Geriatr Psychiatry 2010;18:256265.

23. Helson H DL, Low A, Bauer B, O’Neil M, Kansagara D, Teo A. Systematic Review of Suicide Prevention in Veterans. VA ESP Project \#05-225 2015.

24. Bolton JM, Spiwak R, Sareen J. Predicting suicide attempts with the SAD PERSONS scale: a longitudinal analysis. J Clin Psychiatry 2012;73:e735e741.

25. Yaseen ZS, Gilmer E, Modi J, Cohen LJ, Galynker, II. Emergency room validation of the revised Suicide Trigger Scale (STS-3): a measure of a hypothesized suicide trigger state. PLoS One 2012;7:e45157.

26. Ono Y, Tanaka E, Oyama H, Toyokawa K, Koizumi T, Shinohe K, et al. Epidemiology of suicidal ideation and help-seeking behaviors among the elderly in Japan. Psychiatry Clin Neurosci 2001;55:605-610.

27. O'Connor E, Gaynes B, Burda BU, Williams C, Whitlock EP. U.S. Preventive Services Task Force Evidence Syntheses, formerly Systematic Evidence Reviews. Screening for Suicide Risk in Primary Care: A Systematic Evidence Review for the US Preventive Services Task Force. Rockville (MD): Agency for Healthcare Research and Quality (US); 2013.

28. Ju YJ, Park EC, Han KT, Choi JW, Kim JL, Cho KH, et al. Low socioeconomic status and suicidal ideation among elderly individuals. Int Psychogeriatr 2016;28:2055-2066.

29. Cho M, Park J, Bae A, Bae JN, Ahn JH, Lee DW, et al. The Epidemiological Survey of Mental Disorder in Korea. Sejong: Minstry of Health \& Welfare, the Republic of Korea; 2011.

30. Nock MK, Borges G, Bromet EJ, Alonso J, Angermeyer M, Beautrais A, et al. Cross-national prevalence and risk factors for suicidal ideation, plans and attempts. Br J Psychiatry 2008;192:98-105. 